\title{
Periods of $\beta$-expansions and linear recurrent sequences
}

\author{
by \\ YAN-Hui Qu, Hui RaO and Ya-Min Yang (Beijing)
}

\section{Introduction}

1.1. $\beta$-numeration system. Let $\beta>1$ be a real number. The $\beta$-transformation is a piecewise linear transformation on $[0,1)$ defined by

$$
T_{\beta}: x \mapsto \beta x-\lfloor\beta x\rfloor,
$$

where $\lfloor\alpha\rfloor$ is the largest integer not exceeding $\alpha$. By iterating this map and considering its trajectory

$$
x \stackrel{x_{1}}{\rightarrow} T_{\beta}(x) \stackrel{x_{2}}{\rightarrow} T_{\beta}^{2}(x) \stackrel{x_{3}}{\rightarrow} \cdots
$$

with $x_{i}=\left\lfloor\beta T_{\beta}^{i-1}(x)\right\rfloor$, we obtain the $\beta$-expansion of $x$,

$$
x=\frac{x_{1}}{\beta}+\frac{x_{2}}{\beta^{2}}+\frac{x_{3}}{\beta^{3}}+\cdots=0 . x_{1} x_{2} x_{3} \ldots
$$

An expansion is finite if $\left(x_{i}\right)_{i \geq 1}$ is eventually 0 . A $\beta$-expansion is periodic if there exists $p \geq 1$ and $M \geq 1$ such that $x_{k}=x_{k+p}$ for all $k \geq M$; if $x_{k}=x_{k+p}$ for all $k \geq 1$, then it is strictly periodic (or purely periodic). When the $\beta$-expansion of $x$ is periodic, we denote by $L_{\beta}(x)$ its minimal period.

When $\beta=b \geq 2$ is an integer, the $\beta$-expansion is the $b$-adic expansion. In this case, it is well known that:

(i) A number $x \in[0,1)$ has periodic expansion if and only if $x$ is a rational number.

(ii) The expansion of $x=p / q$ is strictly periodic if and only if $\operatorname{gcd}\{q, b\}$ $=1$.

(iii) The minimum period of the b-adic expansion of $p / q$ coincides with the minimum period of the sequence $\left\{b^{n}(\bmod q)\right\}_{n \geq 0}$. 
It is natural to ask the same questions for $\beta$-expansions when $\beta$ is not an integer.

(Q1) For which $x \in[0,1)$, is the $\beta$-expansion periodic?

(Q2) For which $x \in[0,1)$, is the $\beta$-expansion strictly periodic?

(Q3) If the $\beta$-expansion of $x$ is periodic, what is the minimum period?

1.2. Periodic $\beta$-expansions and Pisot numbers. Question (Q1) has been settled for all Pisot numbers (see Schmidt [Sch], 1980). An algebraic integer strictly greater than 1 is a Pisot number if all its algebraic conjugates have modulus strictly less than 1 . A number is called a Pisot unit if it is a Pisot number and an algebraic unit. Let $\mathbb{Q}(\beta)$ be the smallest field containing the rational numbers and $\beta$. The following is well known:

TheOREM A (Schmidt [Sch]). Let $\beta$ be a Pisot number and $x \in[0,1)$. Then $x$ has periodic $\beta$-expansion if and only if $x \in \mathbb{Q}(\beta)$.

The $\beta$-expansion with a Pisot base has drawn attention of many mathematicians. For a Pisot unit $\beta$, Rauzy ([Rau], 1982) and Thurston ([Thu], 1989) constructed a self-similar tiling system. The tiles (usually with fractal boundaries) are called Rauzy fractals or atomic surfaces. Several interesting dynamical system are related to Rauzy fractals (cf. [AI, IR2]).

According to the behavior of the $\beta$-expansion, several algebraic properties of $\beta$ have been defined, for example, the $(\mathrm{F})$-property introduced by Frougny and Solomyak ([FS]) and the (W)-property introduced by Akiyama [Aki]. Further investigation of these properties can be found in [Hol] (for the (F)-property) and [ARS] (for the (W)-property).

Akiyama [Aki] showed that the algebraic properties of $\beta$ characterize the tiling and dynamical properties of the associated Rauzy fractals.

On the other hand, Ito and his coauthors ([HI, IS, IR1]) employed the Rauzy fractals to study the $\beta$-expansions. They answered Question (Q2) when $\beta$ is a Pisot unit (see Section 1.3). The goal of this paper is to answer Question (Q3) when $\beta$ is a quadratic Pisot unit.

1.3. Strictly periodic $\beta$-expansions with Pisot unit base. Let $\beta$ be a Pisot unit of degree $d$. By using Rauzy fractals, a region $K$ has been constructed to serve as a Markov partition of a group automorphism on $d$-dimensional torus ([Pra, IR2] $)$. It has been proved that the region $K$ completely characterizes the strictly periodic $\beta$-expansions in base $\beta$. The result was first obtained for quadratic Pisot units in [HI], generalized to a family of Pisot units in [IS], and to all Pisot units in [IR2].

Here we state only the result for quadratic Pisot units, which is needed in the present paper. A number $\beta>1$ is a quadratic Pisot unit if either $\beta^{2}=n \beta+1(n \geq 1)$ or $\beta^{2}=n \beta-1(n \geq 3)$. Since $1, \beta$ is a basis of the field $\mathbb{Q}(\beta)$, each $x \in \mathbb{Q}(\beta)$ can be uniquely expressed as $x=x_{1}+x_{2} \beta$ with 
$x_{1}, x_{2} \in \mathbb{Q}$. The number $x^{\prime}=x_{1}+x_{2} \beta^{\prime}$ is the conjugate of $x$ in $\mathbb{Q}(\beta)$ by definition, where $\beta^{\prime}$ is the algebraic conjugate of $\beta$.

Theorem B ([HI, IS, IR1]). (i) If $\beta>1$ satisfies $\beta^{2}=n \beta+1$ and $x \in[0,1]$ then $x$ has strictly periodic $\beta$-expansion if and only if $x \in \mathbb{Q}(\beta)$ and $\left(x, x^{\prime}\right)$ belongs to the closed set $K$ in Figure 1.a minus the lines $x=1 / \beta$ and $x=1$.

(ii) If $\beta>1$ satisfies $\beta^{2}=n \beta-1$ and $x \in[0,1]$ then $x$ has strictly periodic $\beta$-expansion if and only if $x \in \mathbb{Q}(\beta)$ and $\left(x, x^{\prime}\right)$ belongs to the closed set $K$ in Figure 1.b.

If we use the concept of "weakly admissible", then we have a uniform statement of Theorem B, which is discussed in full detail in Section 2.

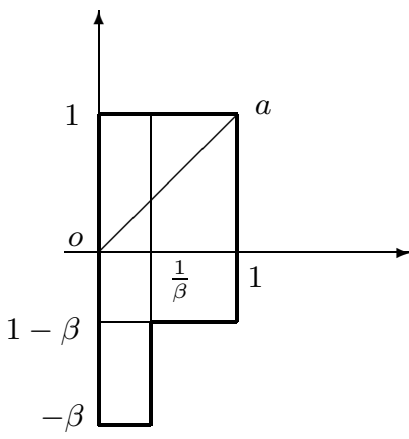

Fig. 1.a

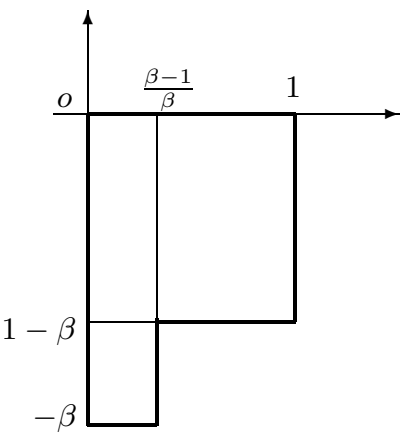

Fig. 1.b

As a consequence, if $\beta^{2}=n \beta+1$, then every rational number in $[0,1)$ has strictly periodic $\beta$-expansion since the segment $o a$ in Figure 1.a is contained in $K$ (this result was first proved by Schmidt [Sch]); if $\beta^{2}=n \beta-1$, then none of the $\beta$-expansions of the rational numbers $\neq 0$ is strictly periodic.

1.4. Periods of $\beta$-expansions with quadratic Pisot unit base. The main purpose of this paper is to investigate the periods of $\beta$-expansions.

When $\beta^{2}=n \beta+1$, the length of the period of the $\beta$-expansion of $p / q$ has been studied by Schmidt [Sch]. He characterized the function $L_{\beta}(p / q)$ by a certain dynamical system.

Using the dynamical and tiling properties of the Rauzy fractal, we obtain a satisfactory answer to Question (Q3) when $\beta$ is a quadratic Pisot unit. Precisely, we show that $L_{\beta}(x)$ is determined by a linear recurrent sequence related to $\beta$ and $x$.

Let $\beta$ be a quadratic Pisot unit with minimal polynomial $P(x)=x^{2}-$ $a_{1} x-a_{0}$ with $a_{0}= \pm 1$. We set

$$
\mathbb{Z}[\beta]:=\left\{c_{0}+c_{1} \beta ; c_{0}, c_{1} \in \mathbb{Z}\right\} .
$$


For $x \in \mathbb{Q}(\beta), x \neq 0$, let $q \geq 1$ be the smallest integer such that $q x \in \mathbb{Z}[\beta]$. Notice that $1, a_{0} \beta^{-1}$ is a basis of $\mathbb{Z}[\beta]$, hence $x$ can be uniquely written as

$$
x=\frac{u_{0}\left(a_{0} \beta^{-1}\right)+u_{1}}{q}
$$

with $u_{0}, u_{1}$ integers and $\operatorname{gcd}\left\{u_{0}, u_{1}, q\right\}=1$.

Let $\left\{u_{k}\right\}$ be the sequence of integers defined by the initial set $u_{0}, u_{1}$ and the recurrence relation

$$
u_{k+1}=a_{1} u_{k}+a_{0} u_{k-1} \quad(k \geq 1) .
$$

We denote this sequence by $u_{k}=u_{k}\left(u_{0}, u_{1}\right)$. It is easy to show that $\left\{u_{k}\right.$ $(\bmod q)\}_{k \geq 0}$ is always strictly periodic $([\mathrm{Eng}, \mathrm{W}])$. It is interesting that this sequence characterizes the periods of $\beta$-expansions.

Theorem 1.1. Let $\beta$ be a quadratic Pisot unit. Suppose $x \neq 0$ and $x=\left(u_{0}\left(a_{0} \beta^{-1}\right)+u_{1}\right) / q$ with $u_{0}, u_{1}, q$ integers and $\operatorname{gcd}\left\{u_{0}, u_{1}, q\right\}=1$. If the $\beta$-expansion of $x$ is strictly periodic, then its periods coincide with the periods of the sequence $\left\{u_{k}\left(u_{0}, u_{1}\right)(\bmod q)\right\}_{k \geq 0}$.

Theorem 1.1 is the main result of this paper. It is proved in Section 2 . The fact that the region $K$ translationally tiles $\mathbb{R}^{2}$ plays a crucial role in our proof (see Figure 2).

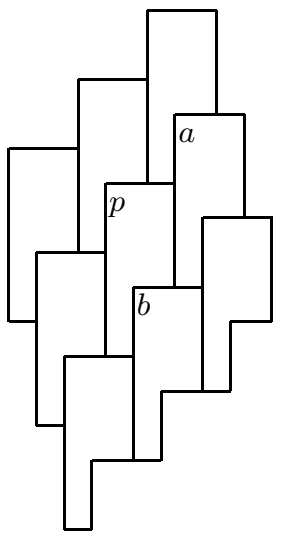

Fig. 2. A tiling by $K$

In the following, we give a second characterization of the function $L_{\beta}(x)$. Denote by $D=a_{1}^{2}+4 a_{0}$ the discriminant of the polynomial $P(x)$. Let $d_{0}$ be the maximal square-free factor of $D$; clearly $\mathbb{Q}(\beta)=\mathbb{Q}\left(\sqrt{d_{0}}\right)$. Let $D_{0}$ be the discriminant of the field $\mathbb{Q}(\beta)$; then $D_{0}=d_{0}$ when $d_{0} \equiv 1(\bmod 4)$ and $D_{0}=4 d_{0}$ otherwise. We note that $I=\sqrt{D / D_{0}}$, the index of the polynomial $P(x)$ in $K$, is an integer. (See for example Hecke [Hec].) 
Let $q \geq 1$ be an integer. Then the sequence $\beta^{k}$ modulo $q$ is strictly periodic since $\beta$ is an algebraic unit. We will prove in Section 3 that this sequence also characterizes the periods of $\beta$-expansions.

Theorem 1.2. Let $\beta$ be a quadratic Pisot unit. Suppose the $\beta$-expansion of $x$ is strictly periodic and $q$ is the smallest integer such that $q x \in \mathbb{Z}[\beta]$. If $q$ is prime to $D / D_{0}$, then the periods of the $\beta$-expansion of $x$ coincide with the periods of the sequence $\left\{q x \beta^{k}(\bmod q)\right\}_{k \geq 0}$.

Let $N(\beta)=\beta \beta^{\prime}=-a_{0}$ denote the norm of $\beta$. When $N(\beta)=-1$, every rational number $p / q$ has strictly periodic $\beta$-expansion. If $q$ is prime to $D / D_{0}$, then the periods coincide with the periods of the sequence $\beta^{k}(\bmod q)$, which is an analogue of the result for $b$-adic expansions.

If $q$ is not prime to $D / D_{0}$, then $L_{\beta}(x)$ is usually a multiple of the minimum period of the sequence $\left\{q x \beta^{k}(\bmod q)\right\}_{k \geq 0}$.

EXAMPLE 1.3. Let $\beta=(1+\sqrt{5}) / 2$ (which satisfies $\beta^{2}=\beta+1$ ) be the golden number. Then every rational number $p / q$ in $[0,1)$ has strictly periodic $\beta$-expansion by Theorem B. According to Theorem 1.1, the minimum period $L_{\beta}(p / q)$ coincides with the minimum period of the sequence $f_{k}$ modulo $q$, where $f_{k}$ is the famous Fibonacci sequence which is defined by

$$
f_{0}=0, \quad f_{1}=1, \quad f_{k+1}=f_{k}+f_{k-1} \quad(k \geq 1) .
$$

The first few terms of the sequence are

$$
0,1,1,2,3,5,8,13,21,34,55,89,144,233,377,610,987, \ldots .
$$

For example, since the sequence $f_{k}$ modulo 7 is

$$
0,1,1,2,3,5,1,6,0,6,6,5,4,2,6,1,0,1, \ldots
$$

and has minimum period 16 , we conclude that $L_{\beta}(1 / 7)=16$. Actually the $\beta$-expansion of $1 / 7$ is $1 / 7=0.000010101010000 \dot{0}$. It is easy to show that $L_{\beta}(p / 7)=16$ for all $1 \leq p \leq 6$.

1.5. Linear recurrent sequences modulo $q$. The periods of linear recurrent sequences modulo $q$ have been studied as early as 1920 by Carmichael [Car] and 1931 by Engstrom [Eng]. By using Dedekind's theorem on decomposition of primes in a number field, Engstrom obtained very general results. Wall [W] (1960) studied the Fibonacci sequence modulo $q$. He obtained many precise and interesting results by a simple method. A complete investigation of linear recurrent sequences of degree 2 modulo $q$ is done in [WY].

2. Proof of Theorem 1.1. In this section, first we investigate the weakly admissible $\beta$-expansions, which is closely related to the boundary 
of the region $K$. Then we show that $K$ can tile $\mathbb{R}^{2}$. Thanks to this tiling property, finally we prove Theorem 1.1.

2.1. Admissible and weakly admissible. Let

$$
\Omega=\left\{x_{1} x_{2} \ldots ; 0 . x_{1} x_{2} \ldots \text { is a } \beta \text {-expansion }\right\} .
$$

We may endow the space $\Omega$ with the discrete metric ([Wal]). The space $\Omega$ is not complete under this metric. Let $\bar{\Omega}$ be the completion of $\Omega$. We will see that $\bar{\Omega}$ consists of weakly admissible sequences.

Formally we may consider the trajectory of 1 :

$$
1 \stackrel{b_{1}}{\rightarrow} T_{\beta}(1) \stackrel{b_{2}}{\rightarrow} T_{\beta}^{2}(1) \stackrel{b_{3}}{\rightarrow} \cdots .
$$

We call $b_{1} b_{2} b_{3} \ldots$ the expansion of 1 and denote it by $d_{\beta}(1)$. Define

$$
d_{\beta}^{*}(1)= \begin{cases}d_{\beta}(1) & \text { if } d_{\beta}(1) \text { is infinite }, \\ \left(b_{1} \ldots b_{d-1}\left(b_{d}-1\right)\right)^{\infty} & \text { if } d_{\beta}(1)=b_{1} \ldots b_{d-1} b_{d} \text { is finite with } b_{d} \neq 0 .\end{cases}
$$

A sequence over the alphabet $\{0,1, \ldots,\lfloor\beta\rfloor\}$ is admissible if starting from any place of the sequence, the right side truncation is lexicographically strictly less than $d_{\beta}^{*}(1)$. If the right side truncations are less than or equal to $d_{\beta}^{*}(1)$, then the sequence is called weakly admissible.

A sequence is a $\beta$-expansion of a certain number if and only if this sequence is admissible. If a $\beta$-expansion is infinite, then it is admissible and weakly admissible. If a $\beta$-expansion of $x$ is finite, then $x$ has another infinite expansion in base $\beta$ which is weakly admissible but not admissible. One can show that $\bar{\Omega}$ is the set of all weakly admissible sequences (cf. [P, Thu]).

Each weakly admissible sequence $\left(x_{i}\right)_{i \geq 1}$ determines a real number $x$, and we call $\left(x_{i}\right)$ the weakly admissible $\beta$-expansion of $x$. Let $\operatorname{Pur}(\beta)$ be the set of numbers in $[0,1)$ with strictly periodic $\beta$-expansions, and let $\operatorname{Pur}^{\prime}(\beta)$ be the set of numbers in $[0,1]$ with strictly periodic, weakly admissible $\beta$ expansions. The difference between $\operatorname{Pur}^{\prime}(\beta)$ and $\operatorname{Pur}(\beta)$ is very small.

TheOREM 2.1. Let $\beta>1$ be a real number. If $d_{\beta}(1)$ is infinite, then $\operatorname{Pur}(\beta)=\operatorname{Pur}^{\prime}(\beta)$; if $d_{\beta}(1)=b_{1} b_{2} \ldots b_{d}$ is finite, then $\operatorname{Pur}^{\prime}(\beta)=\operatorname{Pur}(\beta) \cup$ $\left\{r_{1}, \ldots, r_{d}\right\}$, where $r_{i}=0 . b_{i} \ldots b_{d}$.

Proof. Suppose $x \in \operatorname{Pur}(\beta)$. Then the $\beta$-expansion of $x$ is infinite and thus it is also admissible. This proves that $\operatorname{Pur}(\beta) \subset \operatorname{Pur}^{\prime}(\beta)$.

Suppose $\operatorname{Pur}(\beta) \neq \operatorname{Pur}^{\prime}(\beta)$. Take any $x \in \operatorname{Pur}^{\prime}(\beta) \backslash \operatorname{Pur}(\beta)$. Then the $\beta$-expansion of $x$ is finite, say $x=0 . x_{1} \ldots x_{k}$. The weakly admissible $\beta$ expansion of $x$ is

$$
x=0 . x_{1} x_{2} \ldots\left(x_{k}-1\right) x_{k+1} x_{k+2} \ldots
$$


where $x_{k+1} x_{k+2} \ldots=d_{\beta}^{*}(1)$. Since the above expansion is strictly periodic, we see that $d_{\beta}^{*}(1)$ is strictly periodic. It follows that $d_{\beta}(1)$ is finite, and $x_{1} x_{2} \ldots\left(x_{k}-1\right) x_{k+1} x_{k+2} \ldots$ coincides with a right side truncation of $d_{\beta}^{*}(1)$. These together imply that $x=r_{i}$ for some $i$.

The following theorem is a special case of a result of [IR1].

Theorem B'. Let $\beta$ be a quadratic Pisot unit. Then $x \in \operatorname{Pur}^{\prime}(\beta)$ if and only if $x \in \mathbb{Q}(\beta) \cap[0,1]$ and $\left(x, x^{\prime}\right) \in K$.

If $\beta^{2}=n \beta+1$, then $d_{\beta}(1)=n 1$; if $\beta^{2}=n \beta-1$, then $d_{\beta}(1)=(n-1)(n-2)^{\infty}$. We see that Theorem B in Section 1 follows directly from Theorem $\mathrm{B}^{\prime}$ and Theorem 2.1.

2.2. A tiling by $K$. Let $\beta$ be a quadratic Pisot unit with minimal polynomial $P(x)=x^{2}-a_{1} x-a_{0}$ where $a_{0}= \pm 1$. Set

$$
\mathcal{J}=\left\{\left(x, x^{\prime}\right) ; x \in \mathbb{Z}[\beta]\right\} .
$$

Then $\mathcal{J}$ is a lattice of $\mathbb{R}^{2}$ with a basis $(1,1),\left(\beta, \beta^{\prime}\right)$. Denote by $E^{\circ}$ the interior of a set $E$.

Lemma 2.2. Let $\beta$ be a quadratic Pisot unit, and let $K$ be the region in Figure 1 associated with $\beta$. Then the collection $\{K+v ; v \in \mathcal{J}\}$ is a translation tiling of $\mathbb{R}^{2}$. That is, $\mathbb{R}^{2}=\bigcup_{v \in \mathcal{J}}(K+v)$ and $\left(K+v_{1}\right)^{\circ} \cap\left(K+v_{2}\right)^{\circ}$ $=\emptyset$ whenever $v_{1} \neq v_{2}, v_{1}, v_{2} \in \mathcal{J}$.

Proof. First we note that $1,1 / \beta$ is a basis of $\mathbb{Z}[\beta]$ and thus $(1,1)$, $\left(1 / \beta, 1 / \beta^{\prime}\right)$ is a basis of $\mathcal{J}$ when $N(\beta)=-1$. Likewise $1,1-\beta^{-1}$ is a basis of $\mathbb{Z}[\beta]$ and thus $(1,1),\left(1-\beta^{-1}, 1-\left(\beta^{\prime}\right)^{-1}\right)$ is a basis of $\mathcal{J}$ when $N(\beta)=1$.

From Figure 2 we see clearly that $K$ tiles the plane by translation, and the translation set is a lattice with a basis $\overrightarrow{p a}, \overrightarrow{p b}$. Since $\overrightarrow{p a}=(1,1), \overrightarrow{p b}=$ $\left(1 / \beta, 1 / \beta^{\prime}\right)$ when $N(\beta)=-1$, and $\overrightarrow{p a}=(1,1), \overrightarrow{p b}=\left(1-\beta^{-1}, 1-\left(\beta^{\prime}\right)^{-1}\right)$ when $N(\beta)=1$, we conclude that the translation set is $\mathcal{J}$ and the lemma is proved.

Lemma 2.3. Let $\beta$ be a quadratic Pisot unit. Let $x, y \in \mathbb{Q}(\beta) \cap[0,1)$, $x, y \neq 0$ and $x \neq y$. If $x-y \in \mathbb{Z}[\beta]$, then at most one of $x, y$ has strictly periodic $\beta$-expansion.

Proof. Suppose one of $\left(x, x^{\prime}\right),\left(y, y^{\prime}\right)$, say $\left(x, x^{\prime}\right)$, is an inner point of the region $K$. Since $K+\mathcal{J}$ is a tiling of $\mathbb{R}^{2}$, we infer that $\left(y, y^{\prime}\right)$ is an inner point of the tile $K+\left(y-x,(y-x)^{\prime}\right)$. Hence $\left(y, y^{\prime}\right)$ is not in $K$ and thus the $\beta$-expansion of $y$ is not strictly periodic. The lemma holds in this case.

Now we consider the case that both $\left(x, x^{\prime}\right),\left(y, y^{\prime}\right)$ are on the boundary of $K$. It is easy to check that

$$
\left\{x \in \mathbb{Q}(\beta) ;\left(x, x^{\prime}\right) \in \partial K\right\}= \begin{cases}\left\{0, \beta^{-1}, 1\right\} & \text { when } N(\beta)=-1, \\ \left\{0,1-\beta^{-1}\right\} & \text { when } N(\beta)=1 .\end{cases}
$$


When $N(\beta)=-1$, only 0 belongs to $\operatorname{Pur}(\beta)$, while $\beta^{-1}$ and 1 belong to $\operatorname{Pur}^{\prime}(\beta)$ but not to $\operatorname{Pur}(\beta)$. When $N(\beta)=1$, we have $0,1-\beta^{-1} \in \operatorname{Pur}(\beta)$. So there is at most one $x \neq 0$ such that $\left(x, x^{\prime}\right)$ is on the boundary of $K$ and $x \in \operatorname{Pur}(\beta)$. The lemma is hence proved in this case.

REMARK 2.4. By the above discussion, we see that $\mathbb{Z}[\beta] \cap \operatorname{Pur}(\beta)=\{0\}$ when $N(\beta)=-1$, while $\mathbb{Z}[\beta] \cap \operatorname{Pur}(\beta)=\left\{0,1-\beta^{-1}\right\}$ when $N(\beta)=1$. Since $1-\beta^{-1}=0 .(n-2)^{\infty}$ when $N(\beta)=1$, one checks directly that Theorem 1.1 holds when $q=1$.

2.3. Carry sequence. Let

$$
P(x)=x^{2}-a_{1} x-a_{0}
$$

be the minimal polynomial of $\beta$. Let $q \geq 1$ be the smallest integer such that $q x \in \mathbb{Z}[\beta]$. Then $x$ can be written uniquely as

$$
x=\frac{u_{0}\left(a_{0} \beta^{-1}\right)+u_{1}}{q}
$$

with $u_{0}, u_{1}$ integers and $\operatorname{gcd}\left\{u_{0}, u_{1}, q\right\}=1$.

We define a sequence $\widetilde{u}_{k}$ as follows. Set $\widetilde{u}_{0}=u_{0}, \widetilde{u}_{1}=u_{1}$. Supposing $\widetilde{u}_{0}, \widetilde{u}_{1}, \ldots, \widetilde{u}_{k}$ are defined, we define $\widetilde{u}_{k+1}$ to be the unique integer such that

$$
\widetilde{u}_{k+1} \equiv a_{1} \widetilde{u}_{k}+a_{0} \widetilde{u}_{k-1}(\bmod q), \quad 0 \leq \widetilde{u}_{k}\left(a_{0} \beta^{-1}\right)+\widetilde{u}_{k+1}<q .
$$

Let us call $\left\{\widetilde{u}_{k}\right\}_{k \geq 0}$ the carry sequence of $x$.

The carry sequence was first introduced by Hollander [Hol] in the case $q=1$, i.e., for $x \in \mathbb{Z}[\beta]$; it has been used in [AR, ARS]. Here we generalize it to all $x \in \mathbb{Q}(\beta)$. The carry sequence is closely related to the $\beta$-expansion.

Let $x=0 . x_{1} x_{2} \ldots$ be the $\beta$-expansion of $x$. Then by (2.1)-(2.3), one has

$$
T_{\beta}(x)=\beta x-x_{1}=\frac{\widetilde{u}_{1}\left(a_{0} \beta^{-1}\right)+\left(a_{1} u_{1}+a_{0} u_{0}-x_{1} q\right)}{q}=\frac{\widetilde{u}_{1}\left(a_{0} \beta^{-1}\right)+\widetilde{u}_{2}}{q} .
$$

In general, it is easy to show by induction that

$$
T_{\beta}^{k}(x)=\frac{\widetilde{u}_{k}\left(a_{0} \beta^{-1}\right)+\widetilde{u}_{k+1}}{q} .
$$

Therefore $x$ has strictly periodic $\beta$-expansion if and only if its carry sequence $\widetilde{u}_{k}$ is strictly periodic. Moreover, $L_{\beta}(x)$ equals the minimal period of the sequence $\widetilde{u}_{k}$.

Proof of Theorem 1.1. Let $\left\{u_{k}\left(u_{0}, u_{1}\right)\right\}_{k \geq 0}$ be the linear recurrent sequence defined by (1.1). We claim that

$$
u_{k} \equiv \widetilde{u}_{k}(\bmod q)
$$

for all $k \geq 0$. For suppose (2.4) holds for $1, \ldots, k$; then

$$
\widetilde{u}_{k+1} \equiv a_{1} \widetilde{u}_{k}+a_{0} \widetilde{u}_{k-1} \equiv a_{1} u_{k}+a_{0} u_{k-1}=u_{k+1}(\bmod q) \text {. }
$$


It remains to show that the sequences $\widetilde{u}_{k}$ and $u_{k}(\bmod q)$ have the same periods, which completes the proof of the theorem.

Let $h$ be a period of the sequence $\widetilde{u}_{k}$. Then $\widetilde{u}_{0}=\widetilde{u}_{h}, \widetilde{u}_{1}=\widetilde{u}_{h+1}$. Thus by $(2.4)$, we have $u_{0} \equiv u_{h}, u_{1} \equiv u_{h+1}(\bmod q)$. Therefore $h$ is a period of the sequence $u_{k}$ modulo $q$ since $u_{k+1}$ is completely determined by $u_{k}$ and $u_{k-1}$.

Conversely, suppose $h$ is a period of $u_{k}(\bmod q)$. Again by (2.4), we have $\widetilde{u}_{0} \equiv \widetilde{u}_{h}, \widetilde{u}_{1} \equiv \widetilde{u}_{h+1}(\bmod q)$. Let $y=T_{\beta}^{h}(x)$. Then

$$
x-y=\frac{\left(\widetilde{u}_{0}-\widetilde{u}_{h}\right)\left(a_{0} \beta^{-1}\right)+\left(\widetilde{u}_{1}-\widetilde{u}_{h+1}\right)}{q} \in \mathbb{Z}[\beta] .
$$

Since both $x$ and $y$ have strictly periodic $\beta$-expansions, we have $x=y$ by Lemma 2.3. Hence $h$ is a period of the $\beta$-expansion of $x$ as well as a period of $\widetilde{u}_{k}$.

3. Proof of Theorem 1.2. To prove Theorem 1.2, we need three easy lemmas. Lemma 3.1 gives a formula for the general term of the sequence $u_{k}$ (see for example [Eng, W]).

LEMma 3.1. The general term of $u_{k}$ is given by

$$
u_{k}=c_{1} \beta^{k}+c_{2}\left(\beta^{\prime}\right)^{k}
$$

where

$$
c_{1}=\frac{u_{1}+a_{0} \beta^{-1} u_{0}}{\beta-\beta^{\prime}}, \quad c_{2}=\left(c_{1}\right)^{\prime}=\frac{u_{1}+a_{0}\left(\beta^{\prime}\right)^{-1} u_{0}}{\beta^{\prime}-\beta} .
$$

Proof. One can check that the initial value of (3.1) is $u_{0}, u_{1}$, and $u_{k}$ in (3.1) satisfies the recurrence relation (1.1).

Let $H(q)$ be the minimal period of the sequence $u_{k}$ modulo $q$. Then we have

Lemma 3.2. If $q=p_{1}^{e_{1}} \cdots p_{k}^{e_{k}}$, then

$$
H(q)=\operatorname{lcm}\left\{H\left(p_{1}^{e_{1}}\right), \ldots, H\left(p_{k}^{e_{k}}\right)\right\}
$$

where $\mathrm{lcm}$ denotes the least common multiple.

Lemma 3.2 can be found in [Eng, W]. We leave the easy proof to the reader.

Let $\Re$ be an ideal in $\mathbb{Q}(\beta)$. Denote by $G(x, \Re)$ the minimal period of the sequence $x, x \beta, x \beta^{2}, \ldots$ modulo $\Re$. Then similar to Lemma 3.2, we have

Lemma 3.3. If $q=p_{1}^{e_{1}} \cdots p_{k}^{e_{k}}$, then

$$
G(x, q)=\operatorname{lcm}\left\{G\left(x, p_{1}^{e_{1}}\right), G\left(x, p_{2}^{e_{2}}\right), \ldots, G\left(x, p_{k}^{e_{k}}\right)\right\} .
$$

Theorem 3.4. Let $x_{0}=u_{0}\left(a_{0} \beta^{-1}\right)+u_{1}$. If $q$ is prime to $D / D_{0}$, then the periods of the sequence $\left\{u_{k}(\bmod q)\right\}$ coincide with the periods of the sequence

$$
x_{0}, x_{0} \beta, x_{0} \beta^{2}, \ldots(\bmod q) .
$$


Proof. Since $a_{0}= \pm 1$, it is easy to see that both $\left\{x_{0} \beta^{k}(\bmod q)\right\}$ and $\left\{u_{k}(\bmod q)\right\}$ are strictly periodic.

According to Lemmas 3.2 and 3.3, we need only show that the assertion is valid for $q=p^{m}$ where $p$ is prime to $D / D_{0}$.

Set $x_{k}=u_{k}\left(a_{0} \beta^{-1}\right)+u_{k+1}, k \geq 0$. Then $x_{k+1}=\beta x_{k}$ and so $x_{k}=x_{0} \beta^{k}$. Hence $x_{k} \in \mathbb{Z}[\beta]$ are algebraic integers in $\mathbb{Q}(\beta)$.

Clearly if $h$ is a period of $\left\{u_{k}(\bmod q)\right\}$, then it is a period of $\left\{x_{k}(\bmod q)\right\}$.

Conversely, suppose $h$ is a period of $\left\{x_{k}(\bmod q)\right\}$. Then $x_{0}\left(\beta^{h}-1\right)$ is divisible by $q=p^{m}$ and so the algebraic conjugate $\left(x_{0}\left(\beta^{h}-1\right)\right)^{\prime}$ is also divisible by $p^{m}$. By Lemma 3.1, we have

Hence

$$
u_{k}=\frac{\left(u_{1}+a_{0} \beta^{-1} u_{0}\right) \beta^{k}-\left(u_{1}+a_{0} \beta^{-1} u_{0}\right)^{\prime}\left(\beta^{\prime}\right)^{k}}{\beta-\beta^{\prime}} .
$$

$$
u_{k+h}-u_{k}=\frac{x_{0}\left(\beta^{h}-1\right) \beta^{k}-x_{0}^{\prime}\left(\left(\beta^{\prime}\right)^{h}-1\right)\left(\beta^{\prime}\right)^{k}}{\beta-\beta^{\prime}} .
$$

By the assumption of $h$, the numerator of the right side of (3.3) is divisible by $p^{m}$. Notice that $\beta-\beta^{\prime}=\sqrt{D}=I \sqrt{D_{0}}$.

If $p$ is prime to $D$, then the denominator is prime to $p^{m}$. Hence $u_{k+h}-u_{k}$ is divisible by $p^{m}$ for all $k \geq 0$, and $h$ is a period of $\left\{u_{k}\left(\bmod p^{m}\right)\right\}$.

If $p$ is a factor of $D_{0}$, then $p=\Re^{2}$ where $\Re$ is a prime ideal in $\mathbb{Q}(\beta)$. We divide the discussion into two cases: $p \neq 2$ and $p=2$. Recall that $D_{0}=d_{0}$ or $4 d_{0}$ where $d_{0}$ is square-free.

If $p \neq 2$, then $p^{2} \nmid D_{0}$ and so the power of $\Re$ contained in the denominator of (3.3) is 1 . Since the power of $\Re$ contained in the numerator of (3.3) is at least $2 m$, we find that $\left(u_{k+h}-u_{k}\right) / p^{m-1}$ is an integer and it is divisible by $p$, the norm of $\Re$. Therefore $u_{k+h}-u_{k}$ is divisible by $q=p^{m}$ for all $k \geq 0$.

If $p=2$, then $D_{0}$ is an even number and hence $D_{0}=4 d_{0}$. Moreover $1, \sqrt{d_{0}}$ is a basis of $\mathbb{Q}(\beta)$. Let $x_{0}\left(\beta^{h}-1\right) \beta^{k}=X+Y \sqrt{d_{0}}$. Then $q=2^{m}$ dividing $X+Y \sqrt{d_{0}}$ implies that $2^{m} \mid Y$. Formula (3.3) becomes

$$
u_{k+h}-u_{k}=\frac{2 Y \sqrt{d_{0}}}{\sqrt{D}}=\frac{2 Y \sqrt{d_{0}}}{I \sqrt{D_{0}}}=\frac{2 Y \sqrt{d_{0}}}{2 I \sqrt{d_{0}}}=\frac{Y}{I} .
$$

Since $p=2$ is coprime to $I$, we conclude that $u_{k+h}-u_{k}$ is divisible by $2^{m}$ for all $k \geq 0$. This completes the proof the theorem.

Theorem 1.2 follows immediately from Theorems 1.1 and 3.4.

\section{References}

[Aki] S. Akiyama, On the boundary of self-affine tilings generated by Pisot numbers, J. Math. Soc. Japan 54 (2002), 283-308.

[AR] S. Akiyama and H. Rao, New criteria for canonical number systems, Acta Arith. 111 (2004), 5-25. 
[ARS] S. Akiyama, H. Rao and W. Steiner, A certain finiteness property of Pisot number systems, J. Number Theory 107 (2004), 135-160.

[AI] P. Arnoux and S. Ito, Pisot substitutions and Rauzy fractals, Bull. Belg. Math. Soc. 8 (2001), 181-207.

[Car] R. D. Carmichael, On sequences of integers defined by recurrence relations, Quart. J. Math. 41 (1920), 343-372.

[Eng] H. T. Engstrom, On sequences defined by linear recurrence relations, Trans. Amer. Math. Soc. 33 (1931), 210-218.

[FS] C. Frougny and B. Solomyak, Finite beta-expansions, Ergodic Theory Dynam. Systems 12 (1992), 713-723.

[HI] Y. Hara and S. Ito, On real quadratic fields and periodic expansions, Tokyo J. Math. 12 (1989), 357-370.

[Hec] E. Hecke, Lectures on the Theory of Algebraic Numbers, Grad. Texts in Math. 77, Springer, 1981.

[Hol] M. Hollander, Linear numeration systems, finite beta expansions, and discrete spectrum of substitution dynamical systems, Ph.D. thesis, Univ. of Washington, 1996.

[IR1] S. Ito and H. Rao, Purely periodic $\beta$-expansions with Pisot unit base, Proc. Amer. Math. Soc. 133 (2005), 953-964.

[IR2] - - - Atomic surfaces, tilings and coincidences I. Irreducible case, Israel J. Math., to appear.

[IS] S. Ito and Y. Sano, On periodic $\beta$-expansions of Pisot numbers and Rauzy fractals, Osaka J. Math. 38 (2001), 349-368.

[P] W. Parry, On the $\beta$-expansion of real numbers, Acta Math. Acad. Sci. Hungar. 11 (1960), 401-416.

[Pra] B. Praggastis, Numeration systems and Markov partitions from self-similar tilings, Trans. Amer. Math. Soc. 351 (1999), 3315-3349.

[Rau] G. Rauzy, Nombres algébriques et substitutions, Bull. Soc. Math. France 110 (1982), 147-178.

[Sch] K. Schmidt, On periodic expansions of Pisot numbers and Salem numbers, Bull. London Math. Soc. 12 (1980), 269-278.

[Thu] W. Thurston, Groups, Tilings, and Finite State Automata, AMS Colloq. Lectures, Amer. Math. Soc., Providence, RI, 1989.

[W] D. Wall, Fibonacci series modulo m, Amer. Math. Monthly 67 (1960), 525-532.

[Wal] P. Walters, An Introduction to Ergodic Theory, Grad. Texts in Math. 79, Springer, 1982.

[WY] M. Wu and Y. M. Yang, Linear recurrent sequence modulo q, preprint, 2004.

Department of Mathematics

Tsinghua University

100084 Beijing, China

E-mail: jyh02@mails.tsinghua.edu.cn

hrao@math.tsinghua.edu.cn

nancy_yym@163.com

Received on 24.9.2004

and in revised form on 22.6.2005 